\title{
Cost Analysis of a Two-Unit Cold Standby System Considering Hardware, Software Failures and Inspection with Maximum Repair Time
}

\author{
M.A.W. Mahmoud \\ Department of Mathematics, \\ Faculty of Science, \\ Al-Azhar University, Nasr City (11884),
} Cairo, Egypt

\author{
M.Y. Haggag \\ Department of Mathematics, \\ Faculty of Science, \\ Al-Azhar University, Nasr City (11884),
} Cairo, Egypt

\author{
A.E.B. Abd Elghany \\ Department of Mathematics, \\ Faculty of Engineering, \\ Benha University (Shoubra Branch), \\ Shoubra, Cairo, Egypt
}

\begin{abstract}
The present paper deals with a reliability model of a two unit cold standby system that exposed to two types of failures. In each unit there are hardware and software componentes work together and fail independently. there is a single server who visits the system immediately when required. The server inspects the unit in case of software failure and decides either to repair or replace it. The $\mathrm{h} / \mathrm{w}$ components undergo for repair at their failure and are replaced by new one in case of arriving to maximum repair time. All time distributions are considered to be exponential. Various measures of reliability of the system are obtained using regenerative point technique. Finally simulation study is done to illustrate the results.
\end{abstract}

\section{General Terms}

Stochastic Process

\section{Keywords}

Cold Standby System, Maximum Repair Time, Inspection, Replacement, Profit Gain

\section{INTRODUCTION}

Recently, with technological progress most of the Industrial Systems consist of software subsystems and hardware subsystems. A lot of research [6, 7, 8, 9] that have been implemented in this region have been taken into account hardware subsystem alone. But there are a lot of complex systems, which have the two subsystems together, such as control systems. [2, 11] developed a combined reliability model for the whole system in which software and hardware components work together. [3] formulated reliability models of a computer system with independent failure of software and hardware components.

Furthermore, the continued operation and ageing of these systems gradually reduce their performance and reliability. [1, 10] proved that the reliability of a system can be increased by making replacement of the components by new one when the repair time is too long. [5, 4] discussed stochastically a two unit cold standby system with the concept of periority subject to maximum operation and maximum repair time.
The aim of this paper is to study a two unit cold standby system considering hardware, software failures and inspection with maximum repair times. Explicit expressions for the following reliability measures of the system are obtained:

1. The mean time to system failure (MTSF).

2. Pointwise and steady state availability.

3. Busy periods with repair due to hardware failures or software failures or replacement or inspection.

4. The profit gain of the system, are obtained.

Finally numerical example is presented to illustrate the theoretical results.

\section{ASSUMPTIONS}

1. The system consists of two identical units.

2. Initially one unit is operating and the other is in standby case (cold standby).

3. The online unit suffers two types of failure, namely, software and hardware failure.

4. There is one server man and always available

5. If the operating unit fails with software failure the server man inspects the unit and decides either to repair with some probability or replace it

6. When the online unit exceeds maximum repair time of hardware failure, the server man will replace it.

7. If the operating unit suffers software failure while replacing the other unit so, there is periorty to repair it after replacing the other unit.

8.The distributions of all times are considered to be stochastically independent random variables, each having an exponential distribution.

9.The connected switch is perfect.

10. After repair, the unit is as good as new. 


\section{NOTATIONS}

$E_{0}:$
$E:$
$\bar{E}:$
$F_{i}(t)(i=1,2):$
$G_{i}(t)(i=1,2):$
$q_{i j}(t), Q_{i j}(t):$

$q_{i j}^{(k)}(t), Q_{i j}^{(k)}(t)$ :

$\Pi_{i}(t):$

$m_{i j}:$

$\mu_{i}$ :

$M_{i}(t)$ :

$A V_{i}(t):$

$k(t), K(t)$ :

$m(t), M(t):$

$h(t), H(t)$ :

$\theta, 1-\theta$ :

$\grave{B}_{i}(t)$ :

$C(t)$ :

$U:$

$*$ :

(c):
State of the system at $\mathrm{t}=0$.

Set of regenerative states.

Set of nonregenrative states.

Cdf of hardware and software failures respectively.

Cdf of repair times.

Pdf and Cdf of time for the system transits from regenerative state $S_{i}$ to $S_{j}$.

Pdf and Cdf of time for the system transits from regenerative state $S_{i}$ to $S_{j}$ via the non-regenerative state $E$.

Cdf of time to system failure starting from state $S_{i} \in E$.

Contribution to mean sojourn time in state $S_{i}$, when system transits direct to $S_{j}$.

$\int \mathrm{P}\left[\right.$ System sojourns in state $\mathrm{S}_{i}$

for at least time $t] \mathrm{dt}$

$\mathrm{P}$ [system is up initially in state $S_{i} \in E$

is up at time $t$ without passing through any other regenerative state or returning to

itself through one or more states $\in E]$.

$\mathrm{P}$ [The system is up at time

$\left.t \backslash E_{0}=S_{i} \in E\right]$.

Pdf and Cdf of replacment time.

Pdf and Cdf of maximum repair time.

Pdf and Cdf of inspection time.

probability that the unit goes for repair due to software failure/ probability that the unit will be replaced due to software failure.

$\mathrm{P}$ [server man is busy with repair of hardware, software failures, replacement and inspection respectively at time t starting from state $S_{i} \in$

$E,(\mathrm{i}=1,2,3,4)]$.

The expected profit incurred in $(0, \mathrm{t}]$.

Dummy variable in Laplace transform (LT).

Symbol for LT.

Symbol for convolution of $\mathrm{f}(\mathrm{t})$ and $\mathrm{g}(\mathrm{t})$

is $f(t)$ C $g(t)=\int_{0}^{t} f(x) d G(t-x)$.

\subsection{Symbols for the states of the system}

$\mathrm{O} / \mathrm{S}$ :

Unit in normal mode / in standby mode.

$\mathrm{r}_{1} / \mathrm{r}_{2}$ :

Unit is in failure mode due to hardware failure and under repair /Unit is in failure mode due to software failure and under repair.

$\mathrm{wr}_{1} / \mathrm{wr}_{2}$ : waiting for repair of hardware failure / waiting for repair software failure

repl /insp: Unit under replacement/ Unit under inspection.

Considering these symbols, the system can be in any one of the following states.

$$
\begin{array}{lll}
\mathrm{S}_{0}=(\mathrm{O}, \mathrm{S}) & \mathrm{S}_{1}=\left(\mathrm{O}, \mathrm{r}_{1}\right) & \mathrm{S}_{2}=(\mathrm{O}, \mathrm{insp}) \\
\mathrm{S}_{3}=\left(\mathrm{O}, \mathrm{r}_{2}\right) & \mathrm{S}_{4}=\left(\mathrm{wr}_{2}, \mathrm{r}_{2}\right) & \mathrm{S}_{5}=\left(\mathrm{wr}_{1}, \mathrm{r}_{2}\right) \\
\mathrm{S}_{6}=\left(\mathrm{wr}_{2}, \mathrm{repl}\right) & \mathrm{S}_{7}=(\mathrm{O}, \mathrm{repl}) & \mathrm{S}_{8}=\left(\mathrm{wr}_{2}, \text { insp }\right) \\
\mathrm{S}_{9}=\left(\mathrm{wr}_{1}, \mathrm{r}_{1}\right) & \mathrm{S}_{10}=\left(\mathrm{wr}_{2}, \mathrm{r}_{1}\right) & \mathrm{S}_{11}=\left(\mathrm{wr}_{1}, \text { insp }\right) \\
\mathrm{S}_{12}=\left(\mathrm{wr}_{1}, \mathrm{repl}\right) & &
\end{array}
$$

Up states $: S_{0}, S_{1}, S_{2}, S_{3}$ and $S_{7}$. Down States: $\mathrm{S}_{4}, \mathrm{~S}_{5}, \mathrm{~S}_{6}, \mathrm{~S}_{8}$, $\mathrm{S}_{9}, \mathrm{~S}_{10}, \mathrm{~S}_{11}$ and $\mathrm{S}_{12}$. One can see that all states are regenerative states.

\subsection{Transition Probabilities and Sojourn Times}

It can be observed that the epoch of entry into any of the states $S_{i} \in$ $E$ is regenerative point. Let $T_{1}(\equiv 0), T_{2}, T_{0}, \ldots$ denote the epochs at which the system enters any state $S_{i} \in E$ let $X_{n}$ denote the state Visited at epoch $T_{n}+$, i.e . just after transition at $T_{n} .\left\{X_{n}, T_{n}\right\}$ is a Markov renewal process with state space $E$ and

$Q_{i j}(t)=P\left[X_{n+1}=j, T_{n+1}-T_{n} \leq t \backslash X_{n}=i\right]$, is the semi Markov kernel over E

The transition probability matrix of embedded Markov chain is $\mathrm{P}=\left(\mathrm{p}_{i j}\right)=\left(Q_{i j}(\infty)=Q(\infty)\right)$, With non zero elements.

By probabilistic arguments, the non zero elements $P_{i j}$ are, Transition form $S_{0}$ :

$P_{0,1}=\frac{\lambda}{\lambda+\alpha}$

where $P_{0,1}$ means that the software failure does not occur until time $\mathrm{t}$ and hardware failure occurs at time $\mathrm{t}$.

All other transition probabilities can be explained in the same manner.

$P_{0,2}=\frac{\alpha}{\lambda+\alpha}$

Transition form $S_{1}: P_{1,0}=\frac{a}{\lambda+\alpha+a+m} P_{1,9}=\frac{\lambda}{\lambda+\alpha+a+m}$ $P_{1,10}=\frac{\alpha}{\lambda+\alpha+a+m} P_{1,7}=\frac{m}{\lambda+\alpha+a+m}$

Transition form $S_{2}: P_{2,11}=\frac{\lambda}{\lambda+\alpha+h} P_{2,8}=\frac{\alpha}{\lambda+\alpha+h} P_{2,7}=$ $(1-\theta) \frac{h}{\lambda+\alpha+h} P_{2,3}=(\theta) \frac{h}{\lambda+\alpha+h}$

Transition form $S_{3}: P_{3,5}=\frac{\lambda}{\lambda+\alpha+b} P_{3,4}=\frac{\alpha}{\lambda+\alpha+b} P_{3,0}=\frac{b}{\lambda+\alpha+b}$ Transition form $S_{4}: P_{4,3}=1$

Transition form $S_{5}: P_{5,1}=1$

Transition form $S_{6}: P_{6,3}=1$

Transition form $S_{7}: P_{7,0}=\frac{k}{\lambda+\alpha+k} P_{7,12}=\frac{\lambda}{\lambda+\alpha+k} P_{7,6}=$ $\frac{\alpha}{\lambda+\alpha+k}$

Transition form $S_{8}: P_{8,6}=(1-\theta) P_{8,4}=(\theta)$

Transition form $S_{9}: P_{9,1}=1$

Transition form $S_{10}: P_{10,3}=1$

Transition form $S_{11}: P_{11,5}=(\theta) P_{11,12}=(1-\theta)$

Transition form $S_{12}: P_{12,1}=1$

We can prove that, $P_{0,1}+P_{0,2}=1, P_{1,0}+P_{1,9}+P_{1,10}+P_{1,7}=1$, $P_{2,11}+P_{2,8}+P_{2,7}+P_{2,3}=1, P_{3,0}+P_{3,4}+P_{3,5}=1, P_{4,3}=$ $P_{5,1}=P_{6,3}=P_{9,1}=P_{10,3}=P_{12,1}=1, P_{7,0}+P_{7,12}+P_{7,6}=$ $1, P_{8,6}+P_{8,4}=1, P_{11,5}+P_{11,12}=1$.

The mean sojourn time $\mu_{i}$ in state $S_{i} \in E$ are, $\mu_{0}=\frac{1}{\lambda+\alpha}$, $\mu_{1}=\frac{1}{\lambda+\alpha+a+m}, \mu_{2}=\frac{1}{\lambda+\alpha+h}, \mu_{3}=\frac{1}{\lambda+\alpha+b}, \mu_{4}=\frac{1}{b}$, $\mu_{5}=\frac{1}{b}, \mu_{6}=\frac{1}{k}, \mu_{7}=\frac{1}{\lambda+\alpha+k}, \mu_{8}=\frac{1}{h}, \mu_{9}=\frac{1}{a}, \mu_{10}=\frac{1}{a}$, $\mu_{11}=\frac{1}{h}, \mu_{12}=\frac{1}{k}$.

We can prove that, $\mu_{0}=m_{01}+m_{02}, \mu_{1}=m_{10}+m_{19}+m_{1,10}+$ $m_{17}, \mu_{2}=m_{2,11}+m_{28}+m_{27}+m_{23}, \mu_{3}=m_{35}+m_{34}+m_{30}$, $\mu_{4}=m_{43}, \mu_{5}=m_{51}, \mu_{6}=m_{63}, \mu_{7}=m_{70}+m_{7,12}+m_{76}$, $\mu_{8}=m_{86}+m_{84}, \mu_{9}=m_{91}, \mu_{10}=m_{10,3}, \mu_{11}=m_{11,5}+$ $m_{11,12}, \mu_{12}=m_{12,1}$.

\subsection{MEAN TIME TO SYSTEM FAILURE}

According to the arguments of theory of regenerative processes, we obtain the following equations,

$$
\bar{\Pi}_{0}(t)=\bar{F}_{1}(t) \bar{F}_{2}(t)+q_{01}(t) \Subset \bar{\Pi}_{1}(t)+q_{02}(t) \Subset \bar{\Pi}_{2}(t) .
$$

The above equation can be explained as follows: The left hand side means that the system works until time $t$. The first term in the right hand side means that the hardware failure does not occur until time $t$ and software failure not occur until time $t$. The second term means that the system moves from state $S_{0}$ to state $S_{1}$ at a time less than time $\mathrm{t}$ and the system completes its operation until time $\mathrm{t}$ starting from state $S_{1}$. The third term means that the system moves from 


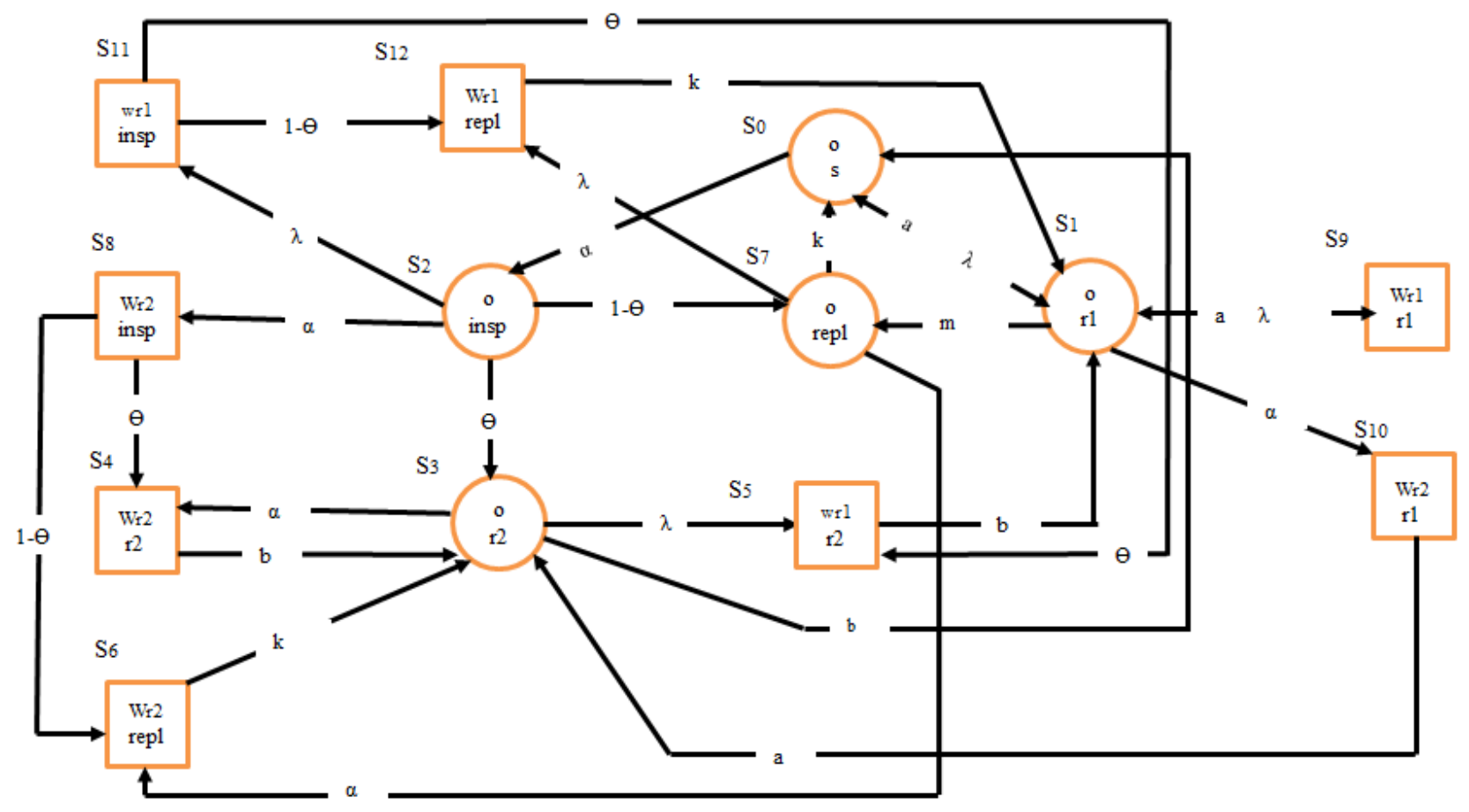

Fig. 1. Transition diagram $\bigcirc$ : Up state, $\square$ : Down state.

state $S_{0}$ to state $S_{2}$ at time less than time t and the system completes its operation until time t starting from state $S_{2}$.

All other equations can be explained in the same manner.

$$
\begin{aligned}
& \bar{\Pi}_{1}(t)=\bar{F}_{1}(t) \bar{F}_{2}(t) \bar{G}_{1}(t) \bar{M}(t)+q_{10}(t) \subset \bar{\Pi}_{1}(t) \\
& +q_{17}(t) \subseteq \bar{\Pi}_{7}(t)
\end{aligned}
$$

$\bar{\Pi}_{2}(t)=\bar{F}_{1}(t) \bar{F}_{2}(t) \bar{H}(t)+q_{23}(t) \Subset \bar{\Pi}_{3}(t)+q_{27}(t) \Subset \bar{\Pi}_{7}(t)$,

$$
\begin{aligned}
& \bar{\Pi}_{3}(t)=\bar{F}_{1}(t) \bar{F}_{2}(t) \bar{G}_{1}(t)+q_{30}(t) \Subset \bar{\Pi}_{0}(t), \\
& \bar{\Pi}_{7}(t)=\bar{F}_{1}(t) \bar{F}_{2}(t) \bar{K}(t)+q_{70}(t) \subsetneq \bar{\Pi}_{0}(t) .
\end{aligned}
$$

Making use of Laplace transform (LT) for Equations [1-5] and solving for $\Pi_{0}^{*}(u)$ considering $\mathrm{u}=0$, we have the time to system failures (MTSF) as follows:

$$
M T S F=E(T)=\frac{l}{L},
$$

where,

$l=\mu_{0}+P_{1}\left(\mu_{1}+P_{1} \mu_{17}\right)+P_{2}\left(\mu_{2}+P_{23} \mu_{3}+P_{27} \mu_{7}\right)$, and

$L=1-P_{1}\left(P_{10}+P_{17} P_{70}\right)-P_{2}\left(P_{23} P_{30}+P_{27} P_{70}\right)$

\section{AVAILABILITY ANALYSES}

Making use of probabilistic arguments, we obtain the following recursive relations for , $\mathrm{i}=0,1,2,3,4,5,6.7,8,9,10,11,12$.
Where

$$
A V_{0}(t)=M_{0}(t)+q_{01}(t)\left(\mathcal{C} A V_{0}(t)+q_{02}(t) \text { (c) } A V_{2}(t),\right.
$$

$$
\begin{aligned}
A V_{1}(t)= & M_{1}(t)+q_{10}(t)\left(\mathcal{C} A V_{0}(t)+q_{17}(t)\left(\mathcal{C} A V_{7}(t)\right.\right. \\
& +q_{1,9}(t)\left(\mathcal{C} A V_{9}(t)+q_{1,10}(t)\left(\mathcal{C} A V_{10}(t),\right.\right.
\end{aligned}
$$

$$
\begin{aligned}
A V_{2}(t)= & M_{2}(t)+q_{23}(t)\left(A V_{3}(t)+q_{28}(t)\left(\mathcal{C} A V_{8}(t)\right.\right. \\
& +q_{27}(t)\left(A V_{7}(t)+q_{2,11}(t)\left(\mathcal{C} A V_{11}(t),\right.\right.
\end{aligned}
$$

$$
\begin{aligned}
A V_{3}(t)= & M_{3}(t)+q_{35}(t)\left(A V_{5}(t)+q_{30}(t)\left(\mathcal{C} A V_{0}(t)\right.\right. \\
& +q_{34}(t)\left(\mathcal{C} A V_{4}(t),\right.
\end{aligned}
$$

$$
A V_{4}(t)=q_{43}(t)\left(\mathcal{C} A V_{3}(t)\right.
$$

$$
A V_{5}(t)=q_{51}(t)\left(\mathcal{C} A V_{1}(t)\right.
$$

$$
A V_{6}(t)=q_{63}(t)\left(\mathcal{C} A V_{3}(t)\right.
$$

$$
\begin{aligned}
A V_{7}(t)= & M_{7}(t)+q_{70}(t), \text { (c) } A V_{0}(t)+q_{7,12}(t) \text { (c) } A V_{12}(t) \\
& +q_{76}(t) \text { (c) } A V_{6}(t),
\end{aligned}
$$

$$
A V_{8}(t)=q_{84}(t) \text { (c) } A V_{4}(t)+q_{86}(t) \text { (c) } A V_{6}(t),
$$




$$
\begin{gathered}
A V_{9}(t)=q_{9,1}(t) \text { (c) } A V_{1}(t), \\
A V_{10}(t)=q_{10,3}(t) \text { (c) } A V_{3}(t),
\end{gathered}
$$

$$
A V_{11}(t)=q_{11,5}(t)\left(\mathcal{C} A V_{5}(t)+q_{11,12}(t) \text { ( }\right) A V_{12}(t),
$$

$$
A V_{12}(t)=q_{12,1}(t) \text { (c) } A V_{1}(t),
$$

where, $M_{0}(t)=e^{-x(\alpha+\lambda)}, M_{1}(t)=e^{-x(\alpha+\lambda+a+m)}, M_{2}(t)=$ $e^{-x(\alpha+\lambda+h)}, M_{3}(t)=e^{-x(\alpha+\lambda+b)}, M_{7}(t)=e^{-x(\alpha+\lambda+k)}$.

Taking LT of [7, 19] to solve them for $A V_{0}^{*}(u)$, then we get the steady state availability of the system $A V_{0}$ in the form,

$$
A V_{0}=\lim _{u \rightarrow 0} u A V_{0}^{*}(u)=\frac{l_{1}}{L_{1}}
$$

\section{BUSY PERIOD ANALYSIS}

\subsection{Expected Busy Period with Repair due to hardware failure:}

Using probabilistic arguments we obtain

$$
\begin{aligned}
& B_{0}^{1}(t)=q_{01}(t) \text { (c) } A V_{0}(t)+q_{02}(t)\left(\mathcal{C} A V_{2}(t),\right. \\
& B_{1}^{1}(t)=\bar{G}_{1}(t)+q_{10}(t) \text { (c) } A V_{0}(t)+q_{17}(t) \text { (c) } A V_{7}(t) \\
& +q_{1,10}(t) \text { (c) } A V_{10}(t)+q_{1,9}(t)\left(\mathcal{C} A V_{9}(t)\right. \text {, } \\
& B_{2}^{1}(t)=q_{23}(t)\left(\mathcal{C} A V_{3}(t)+q_{28}(t)(\mathrm{C}) A V_{8}(t)+q_{27}(t)\left(\mathrm{C} A V_{7}(t)\right.\right. \\
& +q_{2,11}(t) \text { (c) } A V_{11}(t)
\end{aligned}
$$

$B_{3}^{1}(t)=q_{35}(t)\left(\mathcal{C} A V_{5}(t)+q_{30}(t)(\mathrm{C}) A V_{0}(t)+q_{34}(t)(\mathrm{C}) A V_{4}(t)\right.$,

$$
\begin{aligned}
& B_{4}^{1}(t)=q_{43}(t) \subseteq A V_{3}(t), \\
& B_{5}^{1}(t)=q_{51}(t) \subseteq A V_{1}(t), \\
& B_{6}^{1}(t)=q_{63}(t)\left(A V_{3}(t),\right.
\end{aligned}
$$

$B_{7}^{1}(t)=q_{70}(t)\left(\mathcal{C} A V_{0}(t)+q_{7,12}(t)\right.$ (c) $A V_{12}(t)+q_{76}(t)$ (c) $A V_{6}(t)$,

$$
\begin{gathered}
B_{8}^{1}(t)=q_{84}(t)\left(A V_{4}(t)+q_{86}(t)\left(A V_{6}(t),\right.\right. \\
B_{9}^{1}(t)=\bar{G}_{1}(t)+q_{9,1}(t)\left(A V_{1}(t),\right.
\end{gathered}
$$

$$
\begin{gathered}
B_{10}^{1}(t)=\bar{G}_{1}(t)+q_{10,3}(t)\left(\mathcal{c} A V_{3}(t),\right. \\
B_{11}^{1}(t)=q_{11,5}(t)\left(\mathcal{C} A V_{5}(t)+q_{11,12}(t)\left(\mathcal{C} A V_{12}(t),\right.\right. \\
B_{12}^{1}(t)=q_{12,1}(t) \text { ( } A V_{1}(t),
\end{gathered}
$$

Using LT to solve equations [21-33] for $B_{0}^{*}(u)$, we have the expected busy period with repair due to hardware failure in steady state as follows

$$
B_{0}^{1}=\lim _{u \rightarrow 0} u B_{0}^{*}(u)=\frac{l_{2}}{L_{1}}
$$

\subsection{Expected Busy Period with Repair due to software failure:}

Similarly as in (5.1), we can get the expected busy period with repair due to software failure in the steady state in the form,

$$
B_{0}^{2}=\lim _{u \rightarrow 0} u B_{0}^{*}(u)=\frac{l_{3}}{L_{1}},
$$

\subsection{Expected Busy Period due to Replacment:}

Similarly as in (5.1), we can get the expected busy period due to replacment in the steady state in the form,

$$
B_{0}^{3}=\lim _{u \rightarrow 0} u B_{0}^{*}(u)=\frac{l_{4}}{L_{1}},
$$

\subsection{Expected Busy Period due to Inspection:}

Similarly as in (5.1), we can get the expected busy period due to inspection in the steady state in the form,

$$
B_{0}^{4}=\lim _{u \rightarrow 0} u B_{0}^{*}(u)=\frac{l_{5}}{L_{1}},
$$

where 


$l_{1}(0)=\left|\begin{array}{ccccccccccccc}A_{00} & A_{01} & A_{02} & 0 & 0 & 0 & 0 & 0 & 0 & 0 & 0 & 0 & 0 \\ A_{10} & 1 & 0 & 0 & 0 & 0 & 0 & A_{17} & 0 & A_{19} & A_{110} & 0 & 0 \\ 0 & 0 & 1 & A_{23} & 0 & 0 & 0 & A_{27} & A_{28} & 0 & 0 & A_{211} & 0 \\ A_{30} & 0 & 0 & 1 & A_{34} & A_{35} & 0 & 0 & 0 & 0 & 0 & 0 & 0 \\ 0 & 0 & 0 & 1 & 1 & 0 & 0 & 0 & 0 & 0 & 0 & 0 & 0 \\ 0 & 1 & 0 & 0 & 0 & 1 & 0 & 0 & 0 & 0 & 0 & 0 & 0 \\ 0 & 0 & 0 & 1 & 0 & 0 & 1 & 0 & 0 & 0 & 0 & 0 & 0 \\ A_{70} & 0 & 0 & 0 & 0 & 0 & A_{76} & 1 & 0 & 0 & 0 & 0 & A_{712} \\ 0 & 0 & 0 & 0 & 0 & 0 & 1-\theta & 0 & 1 & 0 & 0 & 0 & 0 \\ 0 & 1 & 0 & 0 & 0 & 0 & 0 & 0 & 0 & 1 & 0 & 0 & 0 \\ 0 & 0 & 0 & 1 & 0 & 0 & 0 & 0 & 0 & 0 & 1 & 0 & 0 \\ 0 & 0 & 0 & 0 & 0 & 0 & 0 & 0 & 0 & 0 & 0 & 1 & 1-\theta \\ 0 & 1 & 0 & 0 & 0 & 0 & 0 & 0 & 0 & 0 & 0 & 0 & 1\end{array}\right|$,

$L_{1}(u)=\left|\begin{array}{ccccccccccccc}1 & B_{01} & B_{02} & 0 & 0 & 0 & 0 & 0 & 0 & 0 & 0 & 0 & 0 \\ B_{10} & 1 & 0 & 0 & 0 & 0 & 0 & B_{17} & 0 & B_{19} & B_{110} & 0 & 0 \\ 0 & 0 & 1 & B_{23} & 0 & 0 & 0 & B_{27} & B_{28} & 0 & 0 & B_{211} & 0 \\ B_{30} & 0 & 0 & 1 & B_{34} & B_{35} & 0 & 0 & 0 & 0 & 0 & 0 & 0 \\ 0 & 0 & 0 & B_{43} & 1 & 0 & 0 & 0 & 0 & 0 & 0 & 0 & 0 \\ 0 & B_{51} & 0 & 0 & 0 & 1 & 0 & 0 & 0 & 0 & 0 & 0 & 0 \\ 0 & 0 & 0 & B_{63} & 0 & 0 & 1 & 0 & 0 & 0 & 0 & 0 & 0 \\ B_{70} & 0 & 0 & 0 & 0 & 0 & B_{76} & 1 & 0 & 0 & 0 & 0 & B_{712} \\ 0 & 0 & 0 & 0 & B_{84} & 0 & B_{86} & 0 & 1 & 0 & 0 & 0 & 0 \\ 0 & B_{91} & 0 & 0 & 0 & 0 & 0 & 0 & 0 & 1 & 0 & 0 & 0 \\ 0 & 0 & 0 & B_{103} & 0 & 0 & 0 & 0 & 0 & 0 & 1 & 0 & 0 \\ 0 & 0 & 0 & 0 & 0 & B_{115} & 0 & 0 & 0 & 0 & 0 & 1 & B_{1112} \\ 0 & B_{121} & 0 & 0 & 0 & 0 & 0 & 0 & 0 & 0 & 0 & 0 & 1\end{array}\right|$

$l_{2}(0)=\left|\begin{array}{ccccccccccccc}0 & A_{01} & A_{02} & 0 & 0 & 0 & 0 & 0 & 0 & 0 & 0 & 0 & 0 \\ \frac{1}{a} & 1 & 0 & 0 & 0 & 0 & 0 & A_{17} & 0 & A_{19} & A_{110} & 0 & 0 \\ 0 & 0 & 1 & A_{23} & 0 & 0 & 0 & A_{27} & A_{28} & 0 & 0 & A_{211} & 0 \\ 0 & 0 & 0 & 1 & A_{34} & A_{35} & 0 & 0 & 0 & 0 & 0 & 0 & 0 \\ 0 & 0 & 0 & 1 & 1 & 0 & 0 & 0 & 0 & 0 & 0 & 0 & 0 \\ 0 & 1 & 0 & 0 & 0 & 1 & 0 & 0 & 0 & 0 & 0 & 0 & 0 \\ 0 & 0 & 0 & 1 & 0 & 0 & 1 & 0 & 0 & 0 & 0 & 0 & 0 \\ 0 & 0 & 0 & 0 & 0 & 0 & A_{76} & 1 & 0 & 0 & 0 & 0 & A_{712} \\ 0 & 0 & 0 & 0 & \theta & 0 & 1-\theta & 0 & 1 & 0 & 0 & 0 & 0 \\ \frac{1}{a} & 1 & 0 & 0 & 0 & 0 & 0 & 0 & 0 & 1 & 0 & 0 & 0 \\ \frac{1}{a} & 0 & 0 & 1 & 0 & 0 & 0 & 0 & 0 & 0 & 1 & 0 & 0 \\ 0 & 0 & 0 & 0 & 0 & 0 & 0 & 0 & 0 & 0 & 0 & 1 & 1-\theta \\ 0 & 1 & 0 & 0 & 0 & 0 & 0 & 0 & 0 & 0 & 0 & 0 & 1\end{array}\right|$,

$l_{3}(0)=\left|\begin{array}{ccccccccccccc}0 & A_{01} & A_{02} & 0 & 0 & 0 & 0 & 0 & 0 & 0 & 0 & 0 & 0 \\ 0 & 1 & 0 & 0 & 0 & 0 & 0 & A_{17} & 0 & A_{19} & A_{110} & 0 & 0 \\ 0 & 0 & 1 & A_{23} & 0 & 0 & 0 & A_{27} & A_{28} & 0 & 0 & A_{211} & 0 \\ \frac{1}{b} & 0 & 0 & 1 & A_{34} & A_{35} & 0 & 0 & 0 & 0 & 0 & 0 & 0 \\ \frac{1}{b} & 0 & 0 & 1 & 1 & 0 & 0 & 0 & 0 & 0 & 0 & 0 & 0 \\ \frac{1}{b} & 1 & 0 & 0 & 0 & 1 & 0 & 0 & 0 & 0 & 0 & 0 & 0 \\ 0 & 0 & 0 & 1 & 0 & 0 & 1 & 0 & 0 & 0 & 0 & 0 & 0 \\ 0 & 0 & 0 & 0 & 0 & 0 & A_{76} & 1 & 0 & 0 & 0 & 0 & A_{712} \\ 0 & 0 & 0 & 0 & \theta & 0 & 1-\theta & 0 & 1 & 0 & 0 & 0 & 0 \\ 0 & 1 & 0 & 0 & 0 & 0 & 0 & 0 & 0 & 1 & 0 & 0 & 0 \\ 0 & 0 & 0 & 1 & 0 & 0 & 0 & 0 & 0 & 0 & 1 & 0 & 0 \\ 0 & 0 & 0 & 0 & 0 & 0 & 0 & 0 & 0 & 0 & 0 & 1 & 1-\theta \\ 0 & 1 & 0 & 0 & 0 & 0 & 0 & 0 & 0 & 0 & 0 & 0 & 1\end{array}\right|$, 


$$
l_{4}(0)=\left|\begin{array}{ccccccccccccc}
0 & A_{01} & A_{02} & 0 & 0 & 0 & 0 & 0 & 0 & 0 & 0 & 0 & 0 \\
0 & 1 & 0 & 0 & 0 & 0 & 0 & A_{17} & 0 & A_{19} & A_{110} & 0 & 0 \\
0 & 0 & 1 & A_{23} & 0 & 0 & 0 & A_{27} & A_{28} & 0 & 0 & A_{211} & 0 \\
0 & 0 & 0 & 1 & A_{34} & A_{35} & 0 & 0 & 0 & 0 & 0 & 0 & 0 \\
0 & 0 & 0 & 1 & 1 & 0 & 0 & 0 & 0 & 0 & 0 & 0 & 0 \\
0 & 1 & 0 & 0 & 0 & 1 & 0 & 0 & 0 & 0 & 0 & 0 & 0 \\
\frac{1}{k} & 0 & 0 & 1 & 0 & 0 & 1 & 0 & 0 & 0 & 0 & 0 & 0 \\
\frac{1}{k} & 0 & 0 & 0 & 0 & 0 & A_{76} & 1 & 0 & 0 & 0 & 0 & A_{712} \\
0 & 0 & 0 & 0 & 0 & 0 & 1-\theta & 0 & 1 & 0 & 0 & 0 & 0 \\
0 & 1 & 0 & 0 & 0 & 0 & 0 & 0 & 0 & 1 & 0 & 0 & 0 \\
0 & 0 & 0 & 1 & 0 & 0 & 0 & 0 & 0 & 0 & 1 & 0 & 0 \\
0 & 0 & 0 & 0 & 0 & 0 & 0 & 0 & 0 & 0 & 0 & 1 & 1-\theta \\
\frac{1}{k} & 1 & 0 & 0 & 0 & 0 & 0 & 0 & 0 & 0 & 0 & 0 & 1
\end{array}\right|,
$$

and

$$
l_{5}(0)=\left|\begin{array}{ccccccccccccc}
0 & A_{01} & A_{02} & 0 & 0 & 0 & 0 & 0 & 0 & 0 & 0 & 0 & 0 \\
0 & 1 & 0 & 0 & 0 & 0 & 0 & A_{17} & 0 & A_{19} & A_{110} & 0 & 0 \\
0 & 0 & 1 & A_{23} & 0 & 0 & 0 & A_{27} & A_{28} & 0 & 0 & A_{211} & 0 \\
0 & 0 & 0 & 1 & A_{34} & A_{35} & 0 & 0 & 0 & 0 & 0 & 0 & 0 \\
\frac{1}{h} & 0 & 0 & 1 & 1 & 0 & 0 & 0 & 0 & 0 & 0 & 0 & 0 \\
0 & 1 & 0 & 0 & 0 & 1 & 0 & 0 & 0 & 0 & 0 & 0 & 0 \\
0 & 0 & 0 & 1 & 0 & 0 & 1 & 0 & 0 & 0 & 0 & 0 & 0 \\
0 & 0 & 0 & 0 & 0 & 0 & A_{76} & 1 & 0 & 0 & 0 & 0 & A_{712} \\
\frac{1}{h} & 0 & 0 & 0 & 0 & 0 & 1-\theta & 0 & 1 & 0 & 0 & 0 & 0 \\
0 & 1 & 0 & 0 & 0 & 0 & 0 & 0 & 0 & 1 & 0 & 0 & 0 \\
0 & 0 & 0 & 1 & 0 & 0 & 0 & 0 & 0 & 0 & 1 & 0 & 0 \\
\frac{1}{h} & 0 & 0 & 0 & 0 & 0 & 0 & 0 & 0 & 0 & 0 & 1 & 1-\theta \\
0 & 1 & 0 & 0 & 0 & 0 & 0 & 0 & 0 & 0 & 0 & 0 & 1
\end{array}\right| .
$$

\section{COST BENEFIT ANALYSES}

where,

$$
\begin{array}{ll}
A_{00}=\frac{1}{\lambda+\alpha}, & A_{10}=\frac{1}{\lambda+\alpha+a+m}, \\
A_{30}=\frac{1}{\lambda+\alpha+b}, & A_{70}=\frac{1}{\lambda+\alpha+k}, \\
A_{01}=\frac{-\lambda}{\lambda+\alpha}, & A_{02}=\frac{-\alpha}{\lambda+\alpha}, \\
A_{19}=\frac{-\lambda}{\lambda+\alpha+a+m}, & A_{110}=\frac{-\alpha}{\lambda+\alpha+a+m}, \\
A_{17}=\frac{-m}{\lambda+\alpha+a+m}, & A_{211}=\frac{-\lambda}{\lambda+\alpha+h}, \\
A_{28}=\frac{-\alpha}{\lambda+\alpha+h}, & A_{27}=(1-\theta) \frac{-h}{\lambda+\alpha+h}, \\
A_{23}=(\theta) \frac{-h}{\lambda+\alpha+h}, & A_{35}=\frac{-\lambda}{\lambda+\alpha+b}, \\
A_{34}=\frac{-\alpha}{\lambda+\alpha+b}, & A_{712}=\frac{-\lambda}{\lambda+\alpha+k}, \\
A_{76}=\frac{-\alpha}{\lambda+\alpha+k}, & \\
\text { and } & \\
B_{01}=-q_{01}^{*}(u), & B_{02}=-q_{02}^{*}(u), \\
B_{10}=-q_{10}^{*}(u), & B_{17}=-q_{17}^{*}(u), \\
B_{19}=-q_{1,9}^{*}(u), & B_{110}=-q_{1,10}^{*}(u), \\
B_{23}=-q_{23}^{*}(u), & B_{27}=-q_{27}^{*}(u), \\
B_{28}=-q_{28}^{*}(u), & B_{211}=-q_{2,11}^{*}(u), \\
B_{30}=-q_{30}^{*}(u), & B_{34}=-q_{34}^{*}(u), \\
B_{35}=-q_{35}^{*}(u), & B_{43}=-q_{43}^{*}(u), \\
B_{51}=-q_{51}^{*}(u), & B_{63}=-q_{63}^{*}(u), \\
B_{70}=-q_{70}^{*}(u), & B_{76}=-q_{76}^{*}(u), \\
B_{712}=-q_{7,12}^{*}(u), & B_{84}=-q_{84}^{*}(u), \\
B_{86}=-q_{86}^{*}(u), & B_{91}=-q_{9,1}^{*}(u), \\
B_{103}=-q_{10,3}^{*}(u), & B_{115}=-q_{11,5}^{*}(u), \\
B_{1112}=-q_{11,12}^{*}(u), & B_{121}=-q_{12,1}^{*}(u) .
\end{array}
$$

Since

$$
C(t)=\varepsilon \mu_{u p}(t)-\sum_{i=1}^{4} K_{i} \mu_{R}^{i}(t)
$$

where,

$\varepsilon:$ revenue per unit of up time

$K_{1}, K_{2}, K_{3}, K_{4}$ : cost per unit time of repair due to hardware, software failures, replacement and inspection respectively.

$$
\begin{gathered}
\mu_{u p}(t)=\int_{0}^{t} A V_{0}(u) d u \\
\mu_{R}^{i}=\int_{0}^{t} B_{0}^{i}(u) d u
\end{gathered}
$$

Using (38), 39) and 40, we have

$$
C^{*}(t)=\varepsilon \mu_{u p}^{*}(t)-\sum_{i=1}^{4} K_{i} \mu_{R}^{i *}(t)
$$

Since the expected profit per unit of time in steady state is,

$$
C=\lim _{t \rightarrow \infty} \frac{C(t)}{t}=\lim _{u \rightarrow 0} u^{2} C^{*}(u)
$$


Then form (42), we get

$$
C=\left(\varepsilon l_{1}-K_{1} l_{2}-K_{2} l_{3}-K_{3} l_{4}-K_{4} l_{5}\right) / L_{1}
$$

where

$\varepsilon:$ is the revenue per unit up-time of the system,

$K_{1}$ : is the cost per unit time for which the server is busy due to hardware failure,

$K_{2}$ : is the cost per unit time for which the server is busy due to software failure,

$K_{3}$ : is the cost per unit time for which the server is busy due to replacement,

$K_{4}$ : is the cost per unit time for which the server is busy due to inspetion.

\section{NUMERICAL EXAMPLE}

By setting $\varepsilon=2000, K_{1}=30, K_{2}=40, K_{3}=100, K_{4}=$ 5 ,figures display the variation of AV, MTSF, and profit respectively, for different values of $\mathrm{a}, \mathrm{b}, \mathrm{k}, \mathrm{m}, \mathrm{h}, \alpha$ and $\lambda$.

\section{$\lambda$}

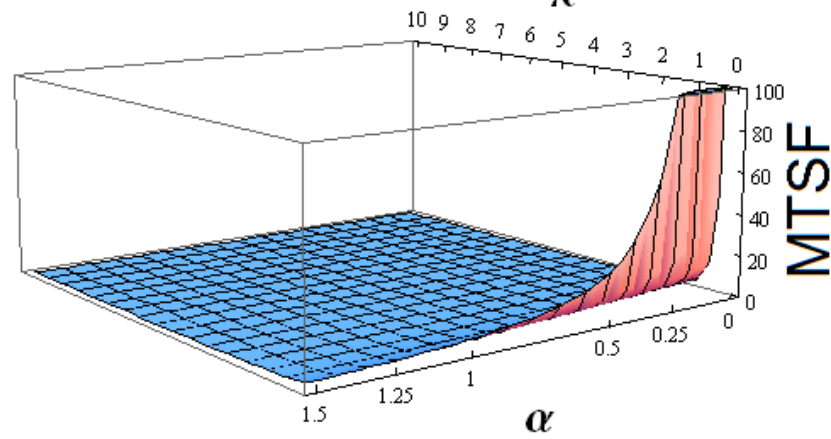

Fig. 2. Variation of Mean Time to System failure (MTSF) with $\alpha, \lambda$ ( $a=$ $2.5, b=5, k=1.2, m=30, h=40, \theta=0.98)$

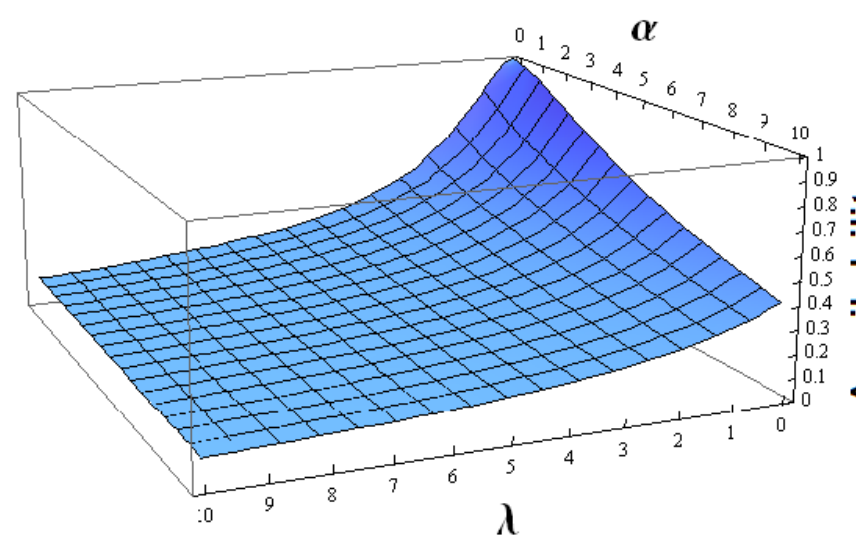

Fig. 3. Variation of Availability (AV) with $\alpha, \lambda(a=2.5, b=5, k=$ $1.2, m=30, h=40, \theta=0.98)$

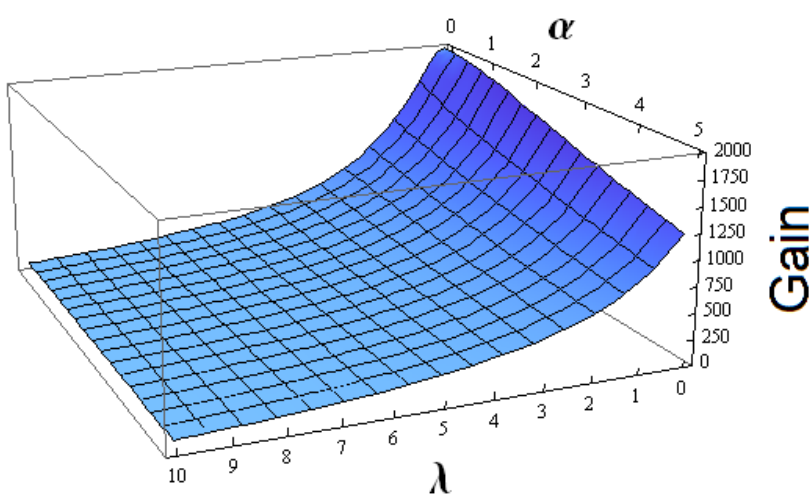

Fig. 4. Variation of Proft Gain (GT) with $\alpha, \lambda,(a=2.5, b=5, k=$ $1.2, m=30, h=40, \theta=0.98)$

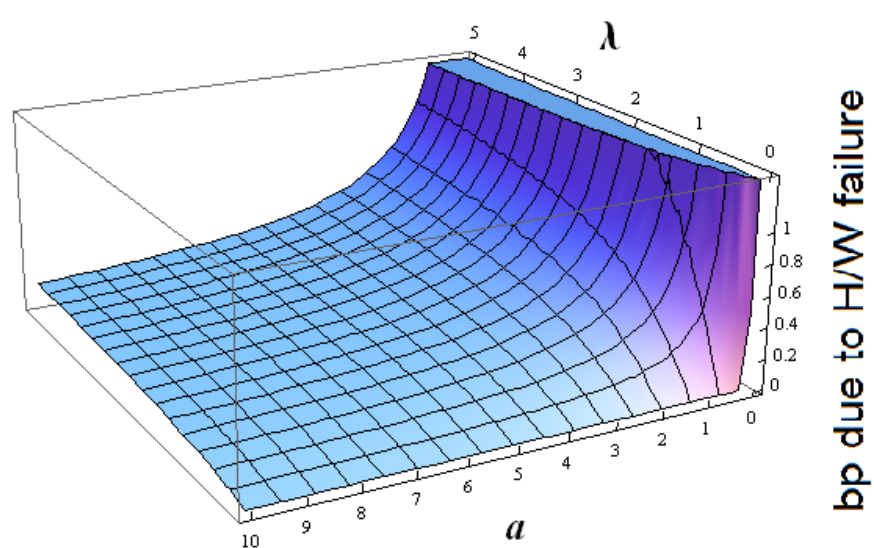

Fig. 5. Variation of Expected Busy Period due to hardware faliure (bp) with $\lambda, a,(b=5, k=1.2, m=30, h=40, \alpha=0.02, \theta=0.98)$

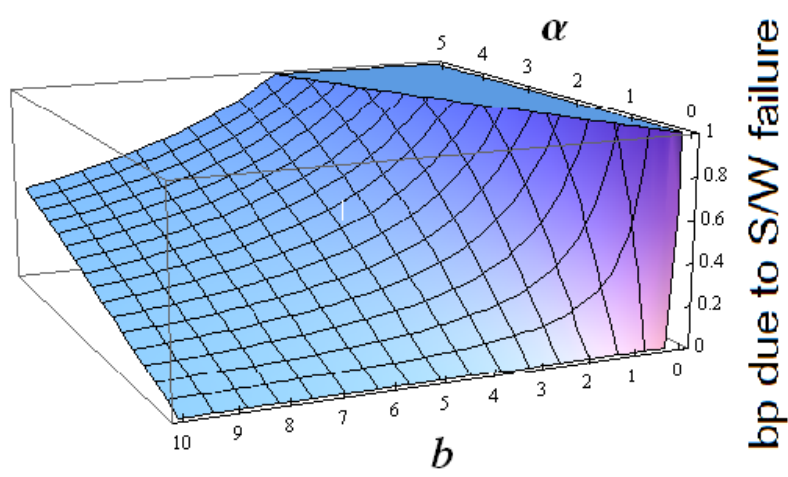

Fig. 6. Variation of Expected Busy Period due to software faliure (bp) with $\alpha, b,(a=2.5, k=1.2, m=30, h=40, \lambda=0.2, \theta=0.98)$ 


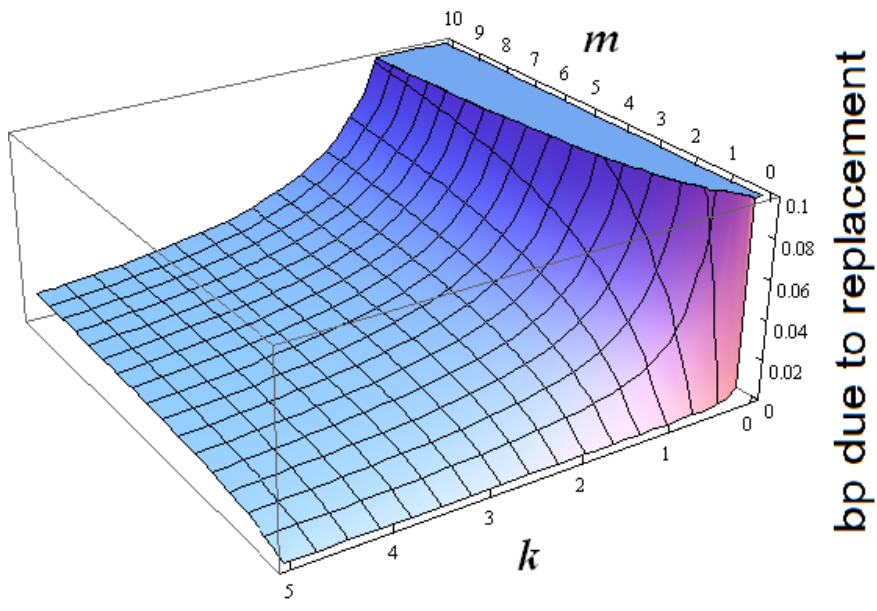

Fig. 7. Variation of Expected Busy Period due to replacement (bp) with $m, k,(a=2.5, b=5, h=40, \lambda=0.1, \alpha=0.02, \theta=0.98)$

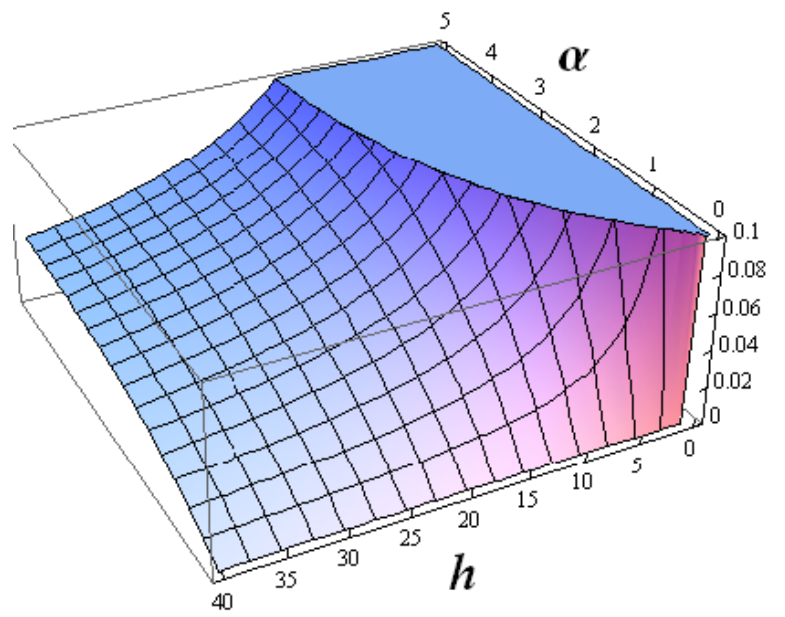

Fig. 8. Variation of Expected Busy Period due to Inspection (bp) with $\alpha, h,(a=2.5, b=5, k=1.2, m=30, \lambda=0.1, \theta=0.98)$

\section{CONCLUSION}

In this paper we successfully obtained some reliability measures of the system such as, the MTSF, the steady state availability, the expected busy periods of the serverman and the expected profit of the system considering hardware failure, software failure, replacement and inspection using regenerative point technique .

1. The MTSF, AV and GT increase with decreasing of $\lambda$.

2. The MTSF, AV and GT increase with decreasing of $\alpha$.

3.The busy period due to $\mathrm{h} / \mathrm{w}$ failure increase with increasing $\lambda$ and decreasing with increasing of $a$.

4.The busy period due to $\mathrm{s} / \mathrm{w}$ failure increase with increasing $\alpha$ and decreasing with increasing of $b$.

5.The busy period due to replacement increase with increasing $m$ and decreasing with increasing of $k$.

6.The busy period due to inspection increase with increasing $\alpha$ and decreasing with increasing of $h$.

According to the results obtained for a particular case, it is concluded that the system model can be more reliable and profitable to use by increasing the repair and inspection rates of the unit and by making immediate replacement of the unit after the completion of maximum repair time.

\section{ACKNOWLEDGMENTS}

The authors would like to thank the referees for their valuable comments that improved the presentation of this article.

\section{REFERENCES}

[1] A. K. Barak and S. C. Malik. Cost-benefit analysis of a computer system with priority to preventive maintenance over hardware repair subject to maximum operation and repair times. Indian Journal of Science and Technology, 6(3):42264234, 2013.

[2] M. A. Friedman and P. Tran. Reliability techniques for combined hardware/software systems. Annual Reliability and Maintability Symposium, pages 290-293, 1992.

[3] A. Kumar and S. C. Malik. Profit analysis of a computer system with priority to software replacement over hardware repair subject to maximum operation and repair times. International Journal of Engineering Science and Technology, 3(10):7452-7468, 2011.

[4] A. Kumar and S. C. Malik. Reliability modeling of a computer system with priority to s/w replacement over $\mathrm{h} / \mathrm{w}$ replacement subject to mot and mrt. International Journal of Pure and Applied Mathematics, 80(5):693-709, 2012.

[5] A. Kumar and S. C. Malik. Stochastic modeling of a computer system with priority to $\mathrm{pm}$ over $\mathrm{s} / \mathrm{w}$ replacement subject to maximum operation and repair times. International Journal of Computer Applications, 43(3):27-34, 2012.

[6] M. A. W. Mahmoud. Probabilistic analysis of a standby system under hardware and human error failures. J. Faculty of Education, Ain Shams University, 20:801-806, 1995.

[7] M. A. W. Mahmoud, M. M. Mohie El Din, and M. E. Moshref. Optimum preventive maintenance for a 2 unit priority standby system with patience time for repair. Optimization, 29:361379, 1994.

[8] M. A. W. Mahmoud and M. A. Esmail. Stochastic analysis of two unit warm standby system with slow switch subject to hardware and human error failures. Microelectron. Reliab, 38:1639-1644, 1998.

[9] M. A. W. Mahmoud and M. E. Moshref. On a two-unit cold standby system considering hardware, human error failures and preventive maintenance. Mathematical and Computer Modelling, 51:736-745, 2010.

[10] S. C. Malik, A. K. Dhankhar, and J. K. Sueria. Stochastic analysis of a system with replacement of unit and server subject to inspection. International Journal of Agricultural and Statistics Sciences, 11(1):81-87, 2015.

[11] S. R. Welke, S. W. Labib, and A. M. Ahmed. Reliability modeling of hardware/software system. IEEE Transactions on Reliability, 44(3):413-418, 1995. 